Artigo

\title{
Morcegos urbanos de Guarulhos: alta riqueza de espécies e dominância de espécies ecologicamente flexíveis reveladas a partir de dados de monitoramento da raiva
}

\author{
Marcos A. Melo',2 (D), David de A. Braga ${ }^{1}$ (1) , Wilson Mansho ${ }^{3}$ (i), Renata R. Carvalho ${ }^{3}$ (D), \\ Débora C. de Oliveira ${ }^{4}$ (i) \& Adriana R. Rosa ${ }^{4}$ (i)
}

\begin{abstract}
1. Seção Técnica de Manejo de Fauna em Vida Livre, Departamento de Conservação de Biodiversidade, Secretaria de Meio Ambiente da Prefeitura de Guarulhos, Av. Dona Glória Pagnoncelli, 344, Guarulhos, SP, Brasil. (mam_melo@yahoo.com.br; davidbraga@gmail.com)

2. Programa de Pós-Graduação em Ecologia e Recursos Naturais, Universidade Federal de São Carlos - UFSCar, Rod. Washington Luiz, Km 235, 13565-905 São Carlos, SP, Brasil.

3. Seção Técnica de Controle de Zoonoses do Centro de Controle de Zoonoses, Departamento de Vigilância em Saúde, Secretaria da Saúde da Prefeitura de Guarulhos. Rua Santa Cruz Descalvados, 420, 07175-370 Guarulhos, SP, Brasil. (wilmansho@hotmail.com; renreinhardt@hotmail.com)

4. Setor de Quirópteros do Centro de Controle de Zoonoses - CCZ, Núcleo de Vigilância, Prevenção e Controle de Fauna Sinantrópica, Coordenação de Vigilância em Saúde - COVISA, Secretaria Municipal de Saúde - SMS, Rua Santa Eulália, 86, São Paulo, SP, Brasil. (dcoliveira@prefeitura.sp.gov.br; arosa@prefeitura.sp.gov.br)
\end{abstract}

Recebido 12 setembro 2019

Aceito 2 abril 2021

Publicado 31 maio 2021

DOI 10.1590/1678-4766e2021009

\begin{abstract}
Urban bats from Guarulhos city: high species richness and dominance of ecologically bats revealed from the rabies monitoring data. Urbanization has impacted bat communities and their ecosystemic services in various regions of the world. However, this impact has been few studied in the Neotropic. In Brazil, although urban bat inventories are scarce, public health organs (e.g., Zoonoses Control Center) perform passive surveillance of infected bats by the rabies virus. These institutions carry out bat identification and collect biologic data essentials to the environment management, population control, ecology, and bat conservation. The present study analyzed bat data (from 2001 to 2017) recorded in Guarulhos city, which integers São Paulo metropolitan region. We compared bat receivement rate, richness, and dominance with other studies. The health surveillance data demonstrated high species richness $(\mathrm{n}=29)$, represented by the three bat families most commonly recorded in Brazilian cities (Phyllostomidae, Molossidadae and Vespertilionidae). Molossus molossus (Pallas, 1766), Glossophaga soricina (Pallas, 1766) and Myotis nigricans (Schinz, 1821) represented most bat data $(70 \%)$ from Guarulhos city. This dominance can be explained by the diet flexibility (insectivores and non-obligate nectarivores) and shelter occupation in buildings of these species, resources highly available in cities. The understood of the urban characteristics associated with urban bat dominance is important by the provide insights on how to mitigate the impacts of urbanization on the richest regional bat community. Thus, it is recommended that cities' environmental planning consider urbanistic and floristic guidelines as a tool integrated for wild life and public health management, and implement strategies to increase the conservation potential in urban ecosystems.
\end{abstract}

KEYWORDS. Chiroptera; urbanization; diet; roosts; buildings.

RESUMO. Urbanização afeta a composição e funções ecossistêmicas das comunidades de morcegos em várias regiões do planeta. Entretanto, este ainda é um tema pouco explorado no Neotrópico. No Brasil, embora poucas cidades apresentem inventários de quirópteros, órgãos municipais de saúde pública (e.g., Centro de Controle de Zoonoses) realizam a vigilância passiva dos morcegos infectados pelo vírus da raiva. Estas instituições fazem a identificação dos espécimes oriundos principalmente de áreas urbanas e coletam dados biológicos importantes para o manejo ambiental, controle populacional, ecologia e conservação das espécies. O presente estudo analisou os dados de recebimento de morcegos, de 2001 a 2017 , oriundos da cidade de Guarulhos, comparando as taxas de recebimento, frequência, riqueza e dominância das espécies com estudos prévios conduzidos em cidades brasileiras. Os dados da vigilância em saúde pública demonstraram uma alta riqueza de espécies $(\mathrm{n}=29)$, representadas por três famílias mais comuns em áreas urbanas brasileiras (Phyllostomidae, Molossidae e Vespertilionidae). Molossus molossus (Pallas, 1766), Glossophaga soricina (Pallas, 1766) e Myotis nigricans (Schinz, 1821) representaram a maioria (70\%) dos dados da quiropterofauna de Guarulhos. Esta dominância pode estar relacionada com a flexibilidade da dieta dessas espécies (insetívoras e nectarívoras não obrigatórias) e abrigos diurnos em edificações, recursos estes altamente disponíveis nas cidades brasileiras. Assim, para mitigar os efeitos da urbanização sobre a rica quiropterofauna regional, recomenda-se que o planejamento ambiental das cidades considere suas diretrizes urbanísticas e florísticas como uma ferramenta ao manejo integrado da fauna silvestre e saúde pública, além de implantar estratégias para aumentar o potencial de conservação da biodiversidade nestes ambientes.

PALAVRAS-CHAVE. Chiroptera; urbanização; dieta; abrigo; edificações.

A urbanização tem conduzido homogeneização biótica das comunidades animais ao redor do planeta, sendo atualmente um dos principais desafios à conservação biológica (Olden et al., 2004; McKinney, 2006; 2008; Devictor et al., 2008). Este efeito se agrava em países em desenvolvimento onde a população humana e as cidades crescem sobre os maiores hotspots de biodiversidade (MYERS et al., 2000) com forte declínio de espécies de aves (SoL et al., 2014) e anfíbios anuros (MACGREGOR-Fors et al., 2012; LOURENÇO-DE-MoRAES et al., 2018). Similarmente, 
urbanização tem afetado negativamente assembleias de morcegos (Chiroptera), substituindo riqueza de espécies por abundância de poucas generalistas (Hourigan et al., 2006; 2010; KunZ et al., 2011), e seus efeitos tem sido pouco estudados em ecossistemas urbanos neotropicais.

Os morcegos exemplificam o importante elo entre o papel ecológico das espécies e a sua inegável contribuição ao bem-estar humano, economia, saúde pública e ambiental (Uieda et al., 1995; Silva et al., 1996; ReIS et al., 2007; Boyles et al., 2011; Almeida et al., 2015). A capacidade de voo e variedade de dietas dos quirópteros faz deles importantes provedores de serviços ecossistêmicos, como a dispersão de sementes, polinização e controle de insetos (REIS et al., 2007). Por outro lado, os morcegos estão envolvidos na epidemiologia de zoonoses importantes como a raiva (Uieda et al., 1995; FAVORETTO et al., 2002; Almeida et al., 2015; CABRAL et al., 2013).

No Brasil, cerca de metade (46\%) das 182 espécies de quirópteros reconhecidas em território nacional já foi registrada em habitats urbanos (NuNES et al., 2016; NOGUEIRA et al., 2018). Nesse ecossistema dominado por humanos, a ampla disponibilidade de abrigos em construções tem favorecido a ocorrência de morcegos generalistas (EVELYN et al., 2004; Almeida et al., 2015), sendo a iluminação pública e arborização viária das cidades fatores responsáveis à atração de morcegos insetívoros e fitófagos, respectivamente (Silva et al., 1996; BREDT et al., 2012). A proximidade de morcegos com diagnóstico positivo para a raiva em áreas com alta densidade de humanos e animais domésticos pode resultar em problemas de saúde pública (De Lucca et al., 2013). Em consequência desse risco epidemiológico nas cidades, a vigilância passiva dos quirópteros infectados pelo vírus da raiva tem sido executada por órgãos municipais de saúde (e.g., Centro de Controle de Zoonozes, CCZ) como parte das ações desenvolvidas nos programas de controle e profilaxia da raiva, implantados em diversas regiões do Brasil (Ministério dA SAÚde, 2005).

Embora o conhecimento da distribuição e ecologia da quiropterofauna urbana brasileira ainda é incipiente (SILVA et al., 1996; PACHECO et al., 2010; Almeida et al., 2015; NuNEs et al., 2016), grande parte das informações provém de programas de vigilância desenvolvidos pelos CCZs municipais. Comumente, esta tem sido a única via de obtenção de dados sobre as comunidades de morcegos registradas em áreas urbanas, pois a insegurança das cidades brasileiras e falta de apoio governamental limitam o desenvolvimento de pesquisas com mamíferos noturnos no país (NUNEs et al., 2016). Dados de ocorrência, distribuição e ecologia das espécies são fundamentais para o planejamento de ações de manejo e conservação dessas espécies, bem como aos programas de educação ambiental e políticas públicas ambientais integradas à saúde. Assim, este estudo teve como objetivo realizar um levantamento da quiropterofauna da área urbana do município de Guarulhos. Especificamente, nosso estudo contribui com informações acerca da distribuição espacial das espécies, taxas de recebimento de morcegos no $\mathrm{CCZ}$, riqueza de espécies, bem como os tipos abrigos diurnos e dietas das espécies registradas. Oportunamente, discutimos de maneira complementar sobre o levantamento prévio de quirópteros das áreas rurais e protegidas da região nordeste deste município (CHAVES et al., 2012), permitindo assim inferir as possíveis causas do recebimento das espécies mais frequentes e comparar com estudos realizados em áreas urbanizadas.

\section{MATERIAL E MÉTODOS}

Área de estudo. O município de Guarulhos $\left(23^{\circ} 28^{\prime} 13^{\prime \prime} \mathrm{S}, 46^{\circ} 31^{\prime} 50^{\prime \prime} \mathrm{W} ; 760 \mathrm{~m}\right.$ a.n.m.; Fig. 1) situase inteiramente na Mata Atlântica, um dos biomas mais ameaçados do mundo (MYERS et al., 2000; FunDAÇÃo SOS Mata AtLÂNTICA \& INPE, 2016). Seu território abrange $318,6 \mathrm{~km}^{2}$, onde vivem 1,3 milhão $(6,3 \%)$ dos 21,2 milhões de habitantes da região metropolitana de São Paulo; a região mais populosa do Brasil (IBGE, 2018). Como consequências da ocupação humana, atualmente restam apenas $27 \%$ das formações florestais originais de Guarulhos (FunDAÇão SOS Mata AtLÂNTICA \& INPE, 2016). Grande parte dos remanescentes florestais está concentrada nas regiões norte e nordeste do município, formando um mosaico de áreas protegidas e não protegidas. O clima é subtropical úmido do tipo $C f b$, oceânico sem estação seca e com verão temperado. A pluviosidade total anual varia de 1,900 a $2,200 \mathrm{~mm}$, e temperatura média anual é de $18-20{ }^{\circ} \mathrm{C}$, de acordo com sistema de Köppen (Alvares et al., 2013).

Coleta de dados. O Centro de Controle de Zoonoses da Secretaria Municipal de Saúde (CCZ-Gru) tem realizado o pronto atendimento das reclamações da população quanto à presença de morcegos na cidade de Guarulhos, sendo esta uma das ações do programa de vigilância e controle da raiva. O serviço oferece orientações técnicas, educativas, manejo ambiental e, havendo a necessidade, ocorre captura/manejo dos morcegos (AlMEIDA et al., 2015). As capturas foram realizadas com auxílio de redes de neblina, puçás, luvas e pinças. Posteriormente os espécimes foram encaminhados para o Setor de Quirópteros do CCZ de São Paulo (CCZ$\mathrm{SP}$ ), onde foram realizados os exames laboratoriais da raiva e a identificação dos espécimes (Almeida et al., 2015). Os indivíduos coletados foram encaminhados com suas respectivas fichas individuais, constando as seguintes informações: data, endereço (convertido a posteriori em latitude e longitude), tipo de coleta, condições de encontro, informações biológicas e dos possíveis abrigos. O mesmo procedimento foi realizado para os animais entregues pelos munícipes ou por outras instituições. Este estudo foi desenvolvido no período de 2001 a 2017. Ressalta-se que, de 2006 a 2008, uma equipe frequentemente realizava a remoção de morcegos em edificações humanas, atendendo uma demanda da população. 

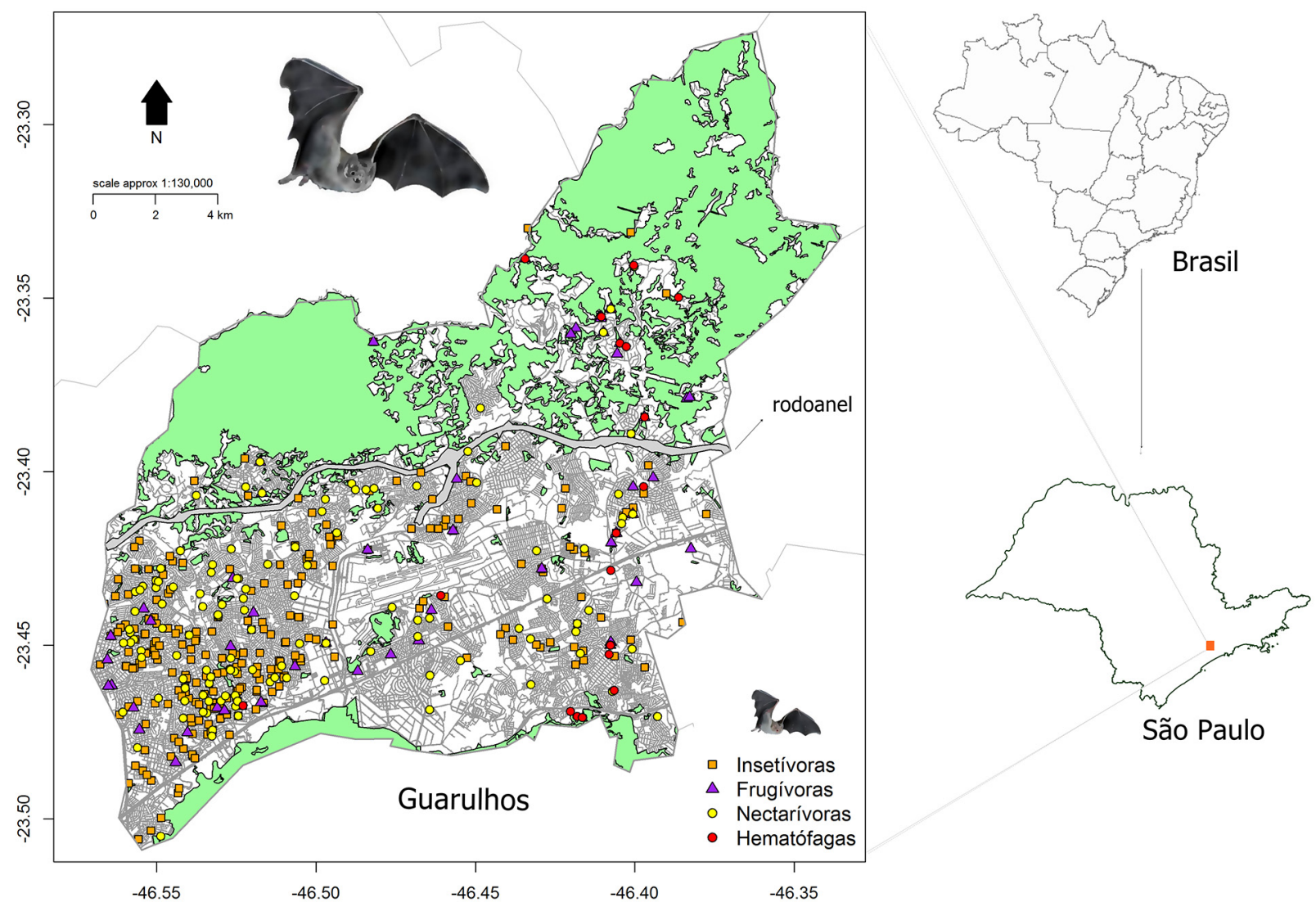

Fig. 1. Distribuição das ocorrências dos morcegos provenientes da cidade de Guarulhos, de acordo com a classificação das espécies em cada categoria trófica. Localização do município de Guarulhos no estado de São Paulo e no Brasil.

Classificações dos dados. As espécies foram identificadas com auxílio de bibliografias disponíveis (Vizotto \& TAdDEI, 1973; Gregorin \& TADDEI, 2002; Gardner 2007; Passos et al., 2010). Os dados foram georreferenciados e plotados em base cartográfica (IBGE, 2010), sendo considerados apenas os registros feitos no território municipal de Guarulhos. Os motivos pelos quais os morcegos geraram ordens de serviços de captura ou como eles foram encontrados na cidade de Guarulhos foram classificados em quatro tipos (Almeida et al., 2015): a) Edificações: espécimes utilizando-se de abrigos artificiais como forros de casas, porões, vãos de dilatação, caixas de persiana, chaminés, garagens, bueiros, dentre outras; b) Atípico: adentramentos ocasionais de morcegos em edificações humanas, incluindo animais encontrados caídos no solo ou enroscados em artefatos antrópicos (e.g., linha de pipa, telas, alambrados, grade de proteção, dentre outros); c) Arborização: captura de morcegos que utilizavam os espécimes arbóreos em áreas urbana/periurbana em busca de alimento, abrigo ou pouso digestório; e d) Outros: morcegos capturados/coletados em áreas verdes do município e que não se enquadram nas categorias anteriores.

A idade e sexo dos espécimes também seguiram os mesmos critérios adotados por AlmEIDA et al. (2015): A, adulto; J, jovem, I, indeterminado; M, macho; F, fêmea. A dieta de cada espécie foi classificada de acordo com BREDT et al. (2012): F, frugívora; H, hematófaga; I, insetívora e $\mathrm{N}$, nectarívora. $\mathrm{O}$ estado de conservação das espécies foi avaliado nas escalas regional (SÃo PAULO, 2018) e global (IUCN, 2019).

Análises de dados. Primeiramente foram calculadas a quantidade total e anual de recebimento de morcegos obtidos na cidade de Guarulhos, bem como a média anual de recebimento. A segunda etapa foi verificar a eficiência do método de coleta de dados ao longo dos anos, com a elaboração da curva de acumulação das espécies considerando o número de espécies recebidas por ano (eixo Y) em função da variável ano (eixo $X, n=17$ ). Para isto, foi utilizada a função "specaccum" do pacote "vegan" com 1,000 permutações (OKSANEN et al., 2017) e excluídos os espécimes não identificados em nível específico. A terceira etapa foi avaliar quais as espécies e famílias que foram mais frequentemente manejadas em Guarulhos, com a execução de um histograma com a quantidade de indivíduos manejados em função das espécies. Além disso, a composição trófica da assembleia de morcegos foi avaliada com base na proporção de espécies e indivíduos classificados em cada item alimentar. Por fim, foram verificados os tipos de ocorrências mais predominantes das espécies registradas em Guarulhos. Para isto, foram excluídos $208(\sim 30 \%)$ registros sem informações quanto ao 
tipo de ocorrência e considerados para esta análise apenas 554 registros que constavam este dado. Para melhor visualização gráfica, foram consideradas apenas as espécies com mais de dez registros. A análise das redes de interações entre as espécies e os tipos de abrigos foi conduzida com auxílio da função "plotweb" do pacote "bipartite" (DoRMAnN et al., 2008).

\section{RESULTADOS}

Dados ecológicos. Um total de 779 indivíduos foi manejado durante o período de 17 anos de estudo; destes, apenas 17 indivíduos não puderam ser determinados em nível de espécie e foram desconsiderados das análises. Assim, 762 indivíduos compuseram um total de 29 espécies (Tab. I). Histiotus velatus (I. Geoffroy, 1824) e Diphylla ecaudata
Spix, 1823 apresentam dados deficientes nos níveis global e regional, respectivamente. Myotis ruber (É. Geoffroy, 1806) e Sturnira tildae de la Torre, 1959 encontram-se regionalmente quase ameaçadas de extinção. A média anual foi 44,8 indivíduos, com o mínimo de exemplares registrados em $2001(\mathrm{n}=5)$ e máximo em $2007(\mathrm{n}=129)$ (Fig. 2). Somente os anos 2004, 2006, 2007 e 2008 ultrapassaram a média anual. A curva de rarefação demonstrou tendência à estabilização (Fig. 3).

As espécies estão distribuídas em três famílias: 39,5\% Phyllostomidae, 37,3\% Molossidae e 23,2\% Vespertilionidae. As espécies mais frequentes foram Molossus molossus (Pallas, 1766), Glossophaga soricina (Pallas, 1766) e Myotis nigricans (Schinz, 1821), representando a maioria (70,7\%) dos morcegos neste estudo (Fig. 4).

Tab. I. Lista das espécies de morcegos identificadas na cidade de Guarulhos, SP, Brasil, segundo o tipo de ocorrência, estado de conservação e dieta [LC, pouco preocupante; NT, quase-ameaçada de extinção; e DD, dados deficientes, segundo IUCN (2019) ${ }^{1}$ e São Paulo (2018) ${ }^{2}$; ver métodos para os detalhes sobre os tipos de ocorrência e dieta. Nectarívora*: espécie nectarívora não obrigatória].

\begin{tabular}{|c|c|c|c|c|c|c|c|}
\hline \multirow[b]{2}{*}{ Família } & \multirow[b]{2}{*}{ Espécie } & \multirow[b]{2}{*}{ Dieta } & \multirow[b]{2}{*}{ Status } & \multicolumn{3}{|c|}{ Tipo de ocorrência } & \multirow[b]{2}{*}{ Outros } \\
\hline & & & & Árvore & Atípico & Edificações & \\
\hline Phyllostomidae & Anoura caudifer & Nectarívora & $\mathrm{LC}$ & 1 & 0 & 4 & 1 \\
\hline Phyllostomidae & Artibeus fimbriatus & Frugívora & $\mathrm{LC}$ & 1 & 1 & 1 & 1 \\
\hline Phyllostomidae & Artibeus lituratus & Frugívora & $\mathrm{LC}$ & 4 & 10 & 9 & 2 \\
\hline Phyllostomidae & Carollia perspicillata & Frugívora & $\mathrm{LC}$ & 0 & 0 & 11 & 0 \\
\hline Molossidae & Cynomops planirostris & Insetívora & $\mathrm{LC}$ & 0 & 0 & 0 & 0 \\
\hline Phyllostomidae & Desmodus rotundus & Hematófoga & $\mathrm{LC}$ & 0 & 2 & 18 & 9 \\
\hline Phyllostomidae & Diphylla ecaudata & Hematófoga & $\mathrm{DD}^{2}$ & 0 & 0 & 0 & 6 \\
\hline Vespertilionidae & Eptesicus brasiliensis & Insetívora & $\mathrm{LC}$ & 0 & 1 & 0 & 0 \\
\hline Vespertilionidae & Eptesicus furinalis & Insetívora & $\mathrm{LC}$ & 0 & 2 & 1 & 1 \\
\hline Molossidae & Eumops auripendulus & Insetívora & $\mathrm{LC}$ & 0 & 3 & 7 & 0 \\
\hline Molossidae & Eumops glaucinus & Insetívora & $\mathrm{LC}$ & 0 & 4 & 2 & 0 \\
\hline Molossidae & Eumops perotis & Insetívora & $\mathrm{LC}$ & 0 & 0 & 2 & 0 \\
\hline Phyllostomidae & Glossophaga soricina & Nectarívora* & $\mathrm{LC}$ & 1 & 41 & 100 & 13 \\
\hline Vespertilionidae & Histiotus velatus & Insetívora & $\mathrm{DD}^{1}$ & 0 & 0 & 0 & 0 \\
\hline Vespertilionidae & Lasiurus cinereus & Insetívora & $\mathrm{LC}$ & 0 & 2 & 0 & 0 \\
\hline Vespertilionidae & Lasiurus ega & Insetívora & $\mathrm{LC}$ & 0 & 4 & 4 & 0 \\
\hline Phyllostomidae & Mimon bennettii & Insetívora & $\mathrm{LC}$ & 0 & 0 & 0 & 1 \\
\hline Molossidae & Molossus molossus & Insetívora & $\mathrm{LC}$ & 3 & 66 & 85 & 8 \\
\hline Molossidae & Molossus rufus & Insetívora & $\mathrm{LC}$ & 0 & 6 & 5 & 0 \\
\hline Vespertilionidae & Myotis nigricans & Insetívora & $\mathrm{LC}$ & 1 & 18 & 55 & 1 \\
\hline Vespertilionidae & Myotis ruber & Insetívora & $\mathrm{NT}^{2}$ & 0 & 1 & 0 & 1 \\
\hline Molossidae & Nyctinomops aurispinosus & Insetívora & $\mathrm{LC}$ & 0 & 1 & 0 & 0 \\
\hline Molossidae & Nyctinomops laticaudatus & Insetívora & $\mathrm{LC}$ & 0 & 1 & 1 & 0 \\
\hline Molossidae & Nyctinomops macrotis & Insetívora & $\mathrm{LC}$ & 0 & 4 & 0 & 0 \\
\hline Phyllostomidae & Platyrrhinus lineatus & Frugívora & $\mathrm{LC}$ & 1 & 2 & 1 & 0 \\
\hline Molossidae & Promops nasutus & Insetívora & $\mathrm{LC}$ & 0 & 2 & 2 & 0 \\
\hline Phyllostomidae & Sturnira lilium & Frugívora & $\mathrm{LC}$ & 4 & 1 & 1 & 0 \\
\hline Phyllostomidae & Sturnira tildae & Frugívora & $\mathrm{NT}^{2}$ & 0 & 0 & 0 & 0 \\
\hline \multirow[t]{2}{*}{ Molossidae } & Tadarida brasiliensis & Insetívora & $\mathrm{LC}$ & 0 & 10 & 1 & 2 \\
\hline & & & Total & 16 & 182 & 310 & 46 \\
\hline
\end{tabular}



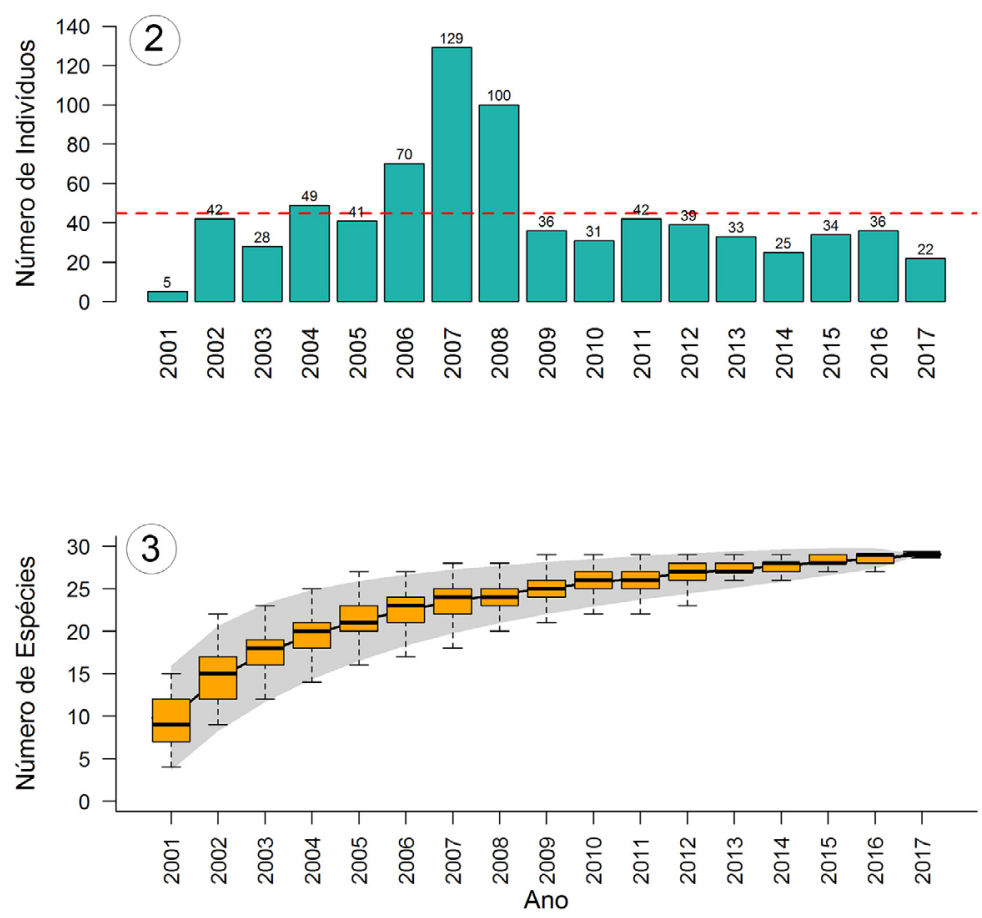

Figs 2, 3. Morcegos identificados na cidade de Guarulhos, SP. Brasil: 2, número de morcegos por ano de 2001 a 2017; 3, Curva de rarefação das espécies [Fig. 2, linha vermelha = média anual de recebimento $(n=44,8)$; Fig. 3, boxplots: representações dos valores anuais de riqueza de espécies; desvios padrões = mancha cinza com 1000 permutações].

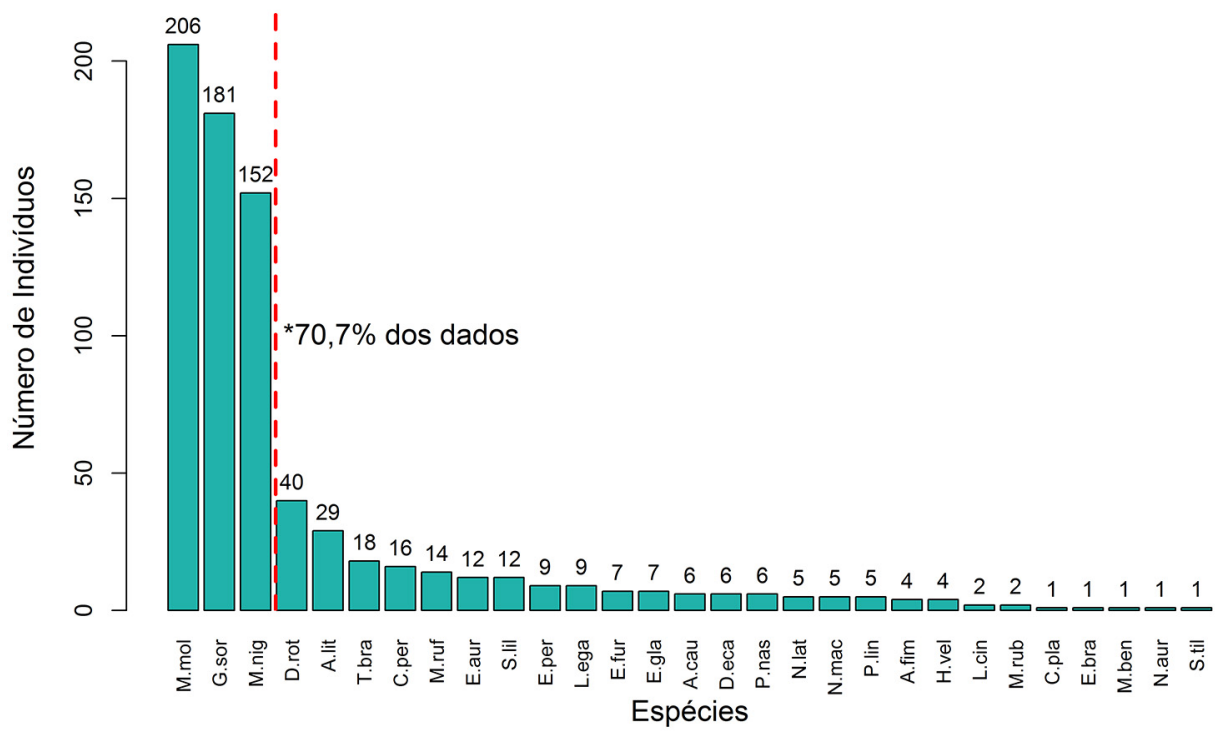

Fig. 4. Quantidade de registros para cada espécie de morcego na cidade de Guarulhos, SP, Brasil. À esquerda da linha vermelha encontram-se as três espécies dominantes (70,7\%) na paisagem urbana de Guarulhos (M.mol, Molossus molossus; G.sor, Glossophaga soricina; M.nig., Myotis nigricans; D.rot, Desmodus rotundus; A.lit, Artibeus lituratus; T.bra, Tadarida brasiliensis; C.per, Carollia pespicillata; M.ruf, M. rufus; E.aur, Eumops auripendulus; S.lil, Sturnira lilium; E.per, E. perotis; L.ega, Lasiurus ega; E.fur, Eptesicus furinalis; E.gla, E. glaucinus; A.cau, Anoura caudifer; D.eca, Dyphilla ecaudatus; P.nas, Promops nasutus; N.lat, Nyctinomops laticaudatus; N.mac, N. macrotis; P.lat, Platyrhinus lineatus; A.fim, A. fimbriatus; H.vel, Histiotus velatus; L.cin, L. cinereus; M.rub, M. ruber; C.pla, Cynomops planirostris; E.bra, E. brasiliensis; M.ben, Mimon bennetti; N.aur, N. aurispinosus e S.til, S. tildae).

Os grupos tróficos com maior riqueza de espécies foram insetívoros $(\mathrm{n}=19 ; 65,5 \%)$ e frugívoros $(\mathrm{n}=6 ; 20,7 \%$; Fig. 5), e a maior abundância foi representada por insetívoros $(\mathrm{n}=462 ; 60,6 \%)$ e nectarívoros $(\mathrm{n}=187 ; 24,55 \%$; Fig. 6$)$.

Dos 554 registros de morcegos com informações sobre o tipo de ocorrência, $56 \%$ deles foram registrados em edificações, seguido de $32,8 \%$ atípico, $8,3 \%$ outros e $2,9 \%$ em árvores. Por outro lado, $35 \%(\mathrm{n}=29)$ das espécies ocorreram no modo atípico, $31,7 \%$ em edificações, $20 \%$ outros e $13,3 \%$ em árvores. Ainda, as três espécies mais frequentes (G. soricina, M. molossus e M. nigricans) também foram as mais registradas em edificações e de forma atípica (Fig. 7). 

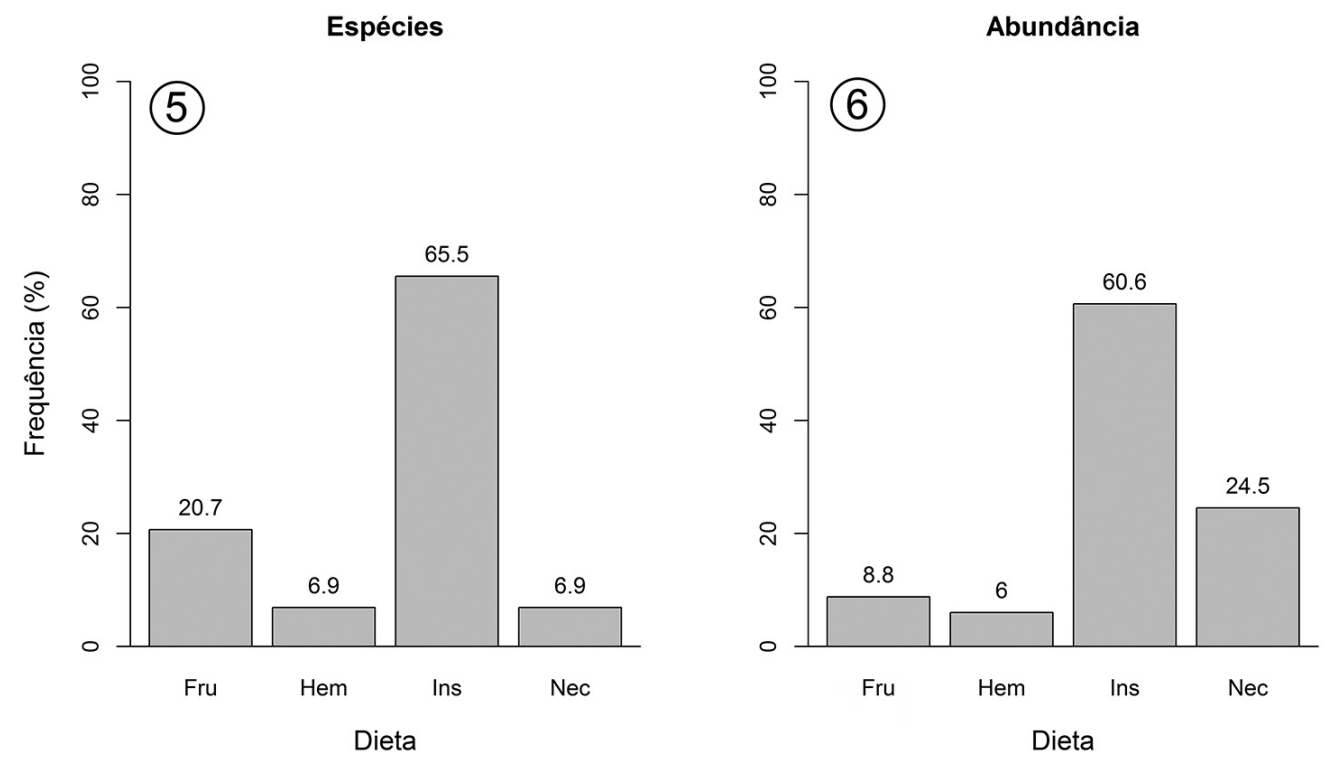

Figs 5, 6. Composição trófica das espécies registradas na cidade de Guarulhos, SP, Brasil: Fig. 5, porcentagens (\%) das espécies (n=29) e Fig. 6, dos (n = 762) indivíduos classificados em cada categoria trófica (Fru, frugívora; Hem, hematófaga; Ins, insetívora; Nec, nectarívora).

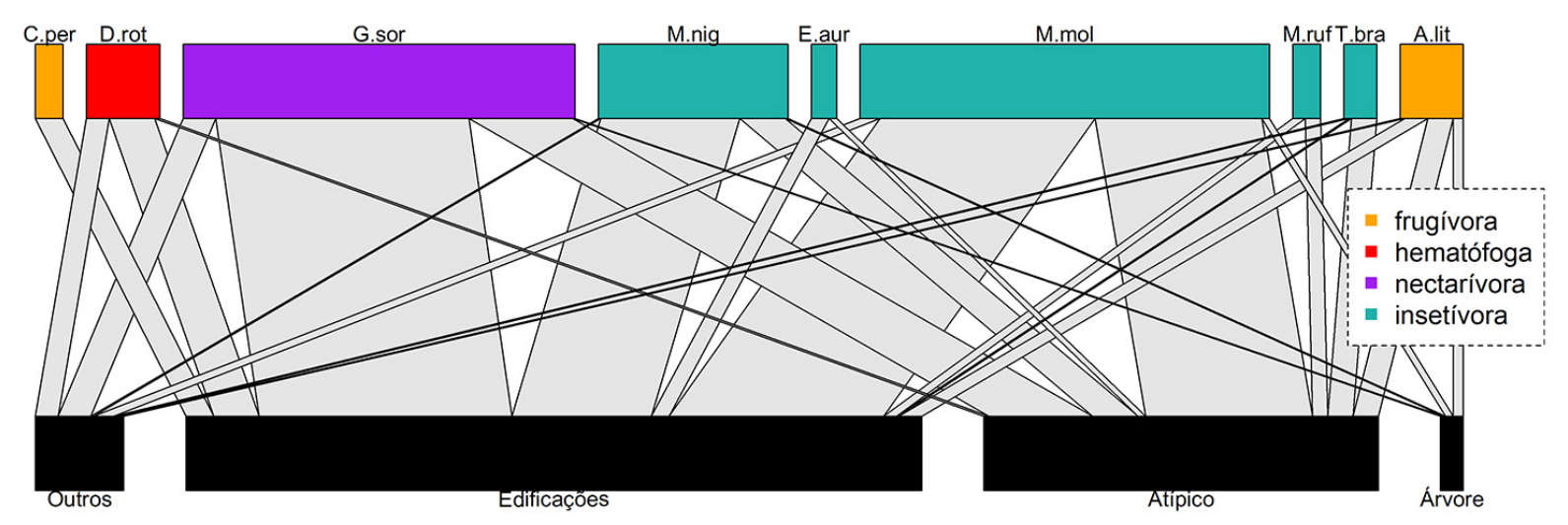

Fig. 7. Redes de interações entre as nove espécies de morcegos mais registradas (acima) classificadas quanto à dieta (legenda) e os tipos de ocorrências/ abrigos (abaixo) de como elas foram encontradas na cidade de Guarulhos, SP, Brasil. Acrônimos das espécies: C.per, Carollia perspicillata; D.rot, Desmodus rotundus; G.sor, Glossophaga soricina; M.nig, Myotis nigricans; E.aur, Eumops auripendulus; M.mol, Molossus molossus; M.ruf, Molossus rufus; T.bra, Tadarida brasiliensis e A.lit, Artibeus lituratus.

\section{DISCUSSÃO}

Contribuições ao conhecimento da quiropterofauna de Guarulhos. O estudo demonstrou uma rica quiropterofauna (29 spp.) registrada nas áreas urbana e periurbana de Guarulhos, a qual compartilha espaço com cerca de 1,3 milhão de habitantes. As espécies estão distribuídas nas três famílias (Phyllostomidae, Molossidae e Vespertilionidae) mais representadas em cidades brasileiras (SILVA et al., 1996; PACHECO et al., 2010; AlMEIDA et al., 2015; NUNES et al., 2016); e as famílias raramente reportadas em áreas urbanas (e.g., Emballonuridae, Mormoopidae e Noctilionidae) mantiveram-se ausentes neste e noutro estudo prévio realizado em áreas rural-florestal deste município (CHAVEs et al., 2012). Há de se destacar que todas as informações sobre as 29 espécies aqui registradas foram exclusivamente provenientes do banco de dados do CCZ. Neste mesmo método, 43 espécies foram registradas na cidade de São Paulo (ALMEIDA et al., 2015), município vizinho à Guarulhos. Entretanto, os números de registros nas duas cidades foram discrepantes. Por exemplo, 762 indivíduos foram identificados durante 17 anos na cidade de Guarulhos, enquanto em São Paulo, 4,248 espécimes foram identificados em 10 anos (ALMEIDA et al., 2015). Considerando que a área territorial do município de São Paulo $\left(1,521 \mathrm{~km}^{2}\right)$ é aproximadamente cinco vezes maior que a de Guarulhos (318 $\left.\mathrm{km}^{2}\right)$ (IBGE, 2018), bem como possui maior número de edificações, deve haver uma possível correlação positiva entre a ocorrência de morcegos e a quantidade de abrigos disponíveis. Além disso, o tempo de atuação do setor de quirópteros e o conhecimento da 
população sobre o serviço prestado pela instituição podem influenciar a taxa de animais coletados e identificados. Na cidade de São Paulo, o serviço prestado pela equipe da prefeitura é referência há mais de 30 anos (SiLVA et al., 1996), enquanto em Guarulhos, iniciou-se 15 anos mais tarde e o atendimento oscilou ao longo do tempo. Por exemplo, em atendimento à demanda da população, uma equipe do CCZ-Gru realizava a remoção de morcegos mais ativamente de 2006 a 2008. Nota-se que estes foram os anos onde a taxa de recebimento de morcegos superou a média, além de 2004. Estas oscilações resultaram nas variações anuais dos valores das coletas de morcegos e ajudam a explicar o menor número de recebimento de morcegos em Guarulhos, quando comparado com o município de São Paulo. De todo modo, a população de Guarulhos (1,3 milhão de habitantes) é cerca de dez vezes menor que a população (12 milhões de habitantes) de São Paulo, com a metade da densidade demográfica $\left(3,834 \mathrm{~km}^{2}\right)$ registrada em São Paulo $(7,398$ hab./ $\mathrm{km}^{2}$ ) (IBGE, 2018). A densidade populacional humana pode reduzir a taxa de encontro/interação entre humanos e animais silvestres (KRESTER et al., 2008) ou não apresentar nenhuma influência (Sousa et al., 2012), mas o aumento na quantidade de reclamações de munícipes ao encontrar animais silvestres está positivamente relacionado com maior escolaridade e poder aquisitivo da população (KOLODINSKY \& ALEONG, 1990), o qual possivelmente tem influenciado na maior taxa de recebimento em São Paulo.

Outros municípios brasileiros tiveram suas quiropterofaunas avaliadas com base em dados provenientes de instituições de saúde e inventários ambientais. Nos municípios situados na região de Cerrado, constam 41 espécies registradas nas cidades de Uberlândia (NunEs et al., 2016) e 28 no Distrito Federal (PACHeCo et al., 2010); na Mata Atlântica do sudeste, registraram-se 48 espécies no município do Rio de Janeiro (NuNES et al., 2016) e 43 na cidade de São Paulo (Almeida et al., 2015); neste mesmo bioma, mas na região sul, PACHECO et al. (2010) relataram 20 e 36 espécies, respectivamente, nas cidades de Curitiba e Porto Alegre. Assim, a compilação dos registros aqui apresentados com os previamente publicados (CHAVES et al., 2012) atualiza a lista de espécies de morcegos de Guarulhos para 40 espécies, representando $22,5 \%$ da quiropterofauna do Brasil (NogueIra et al., 2018) e 50,5\% daquela conhecida para o estado de São Paulo (GARBINo, 2016). Este resultado está em conformidade com as quiropterofaunas mais ricas do sudeste brasileiro, ficando atrás apenas de Uberlândia (41), São Paulo (43) e Rio de Janeiro (48)(Almeida et al., 2015; NunEs et al., 2016). Isto mais uma vez demonstra a importância do monitoramento de morcegos realizado pelos CCZs (ALMEIDA et al., 2015), não apenas para a vigilância em saúde, mas também por ampliar o conhecimento da ocorrência das espécies nos municípios.

Dominância e possíveis fatores relacionados. A compreensão dos fatores que contribuem para a ocorrência das interações entre humanos e fauna silvestre, especialmente aos conflitos com humanos, são essenciais para o manejo efetivo das espécies (SouzA et al., 2012). Urbanização tem levado à alta dominância de poucas espécies de morcegos em diversas cidades do mundo (Russo \& AnCILlotto, 2015). Nossos dados demonstraram uma alta dominância de registros de M. molossus, G. soricina e M. nigricans. No Brasil, essas espécies também foram as mais frequentes nas cidades de São Paulo (Silva et al.,1996; Almeida et al., 2015) e Distrito Federal (BRedt \& Uieda, 1996; PACHeCo et al., 2010), mas as duas últimas foram incomuns em algumas cidades do sul do país (PACHECo et al., 2010).

Algumas espécies apresentam-se tolerantes a uma cidade, mas aversivas a outra. Isto pode ser resultante da seleção de traços ecológicos das espécies mais adaptadas a prosperar em um ecossistema urbano que outra (JuNG \&THRELFALL, 2018) ou apenas refletindo o viés biogeográfico da região das cidades (COLEMAN \& BARCLAY, 2012). O tamanho dos agrupamentos (número de indivíduos) das espécies pode variar conforme a região estudada (PACHECO et al., 2010), mas variações na frequência de indivíduos identificados na mesma mancha urbana e sob o mesmo método de estudo (e.g., Guarulhos e São Paulo) podem indicar certa preferência de abrigos das espécies e/ou alta oferta destes nas edificações humanas da área de estudo. Por exemplo, Tadarida brasiliensis (I. Geoffroy, 1824) foi comum na cidade de São Paulo, mas pouco registrada em Guarulhos. Esta espécie prefere dormitórios grandes $\left(>100 \mathrm{~m}^{2}\right)$ cobertos por telhas de cerâmica (PACHECO et al., 2010) e edifícios altos $(>10 \mathrm{~m})$ e abandonados também fornecem condições favoráveis (BIAVATTI et al., 2015; Li \& WiLKINS, 2015). Embora T. brasiliensis também utilize caixas de persianas nas cidades de Porto Alegre e São Paulo (Pacheco et al., 2010), este comportamento não foi observado no presente estudo. Por outro lado, M. molossus foi proporcionalmente mais frequente em Guarulhos, a qual prefere áreas menores cobertas por telhas de amianto (atualmente fibrocimento) de edificações baixas (PACHECO et al., 2010). Glossophaga soricina tem sido afetada negativamente pela escassez de casas com porões e sótãos no Distrito Federal (BREDT \& UIEDA,1996), mas na região metropolitana de São Paulo, incluindo este estudo, esta é uma das espécies mais frequentes (Silva et al., 1996; Almeida et al., 2015). Contudo, estes últimos autores aventam uma atraente hipótese que $G$. soricina deva ser mais detectada por comumente utilizar garagens e porões, locais estes mais facilmente detectados por humanos. Para testar essa hipótese, sugerimos incluir no banco de dados dos CCZs a informação de como os munícipes observaram as espécies, aliado com características do biótopo de encontro.

A alta frequência de morcegos em edificações na cidade de Guarulhos deve-se a dominância de espécies como M. molossus, M. nigricans e G. soricina, as quais utilizam telhados, forros, garagens e porões como abrigos. Aqui este tipo de ocorrência foi proporcionalmente maior que a encontrada na cidade de São Paulo (AlmeIDA et al., 2015). Estes autores obtiveram maior porcentagem de animais coletados/recebidos em situações atípicas. No presente estudo, situação atípica foi a segunda maior causa de recebimento. Esse dado é importante porque a maioria dos morcegos com 
diagnóstico positivo para a raiva foi encontrada em condições atípicas, oferecendo potencial risco à saúde pública (SILVA et al., 1996; AlmeIDA et al., 2011; 2015). Muito embora este risco seja menor que outras doenças transmitidas por mosquitos e roedores (e.g., dengue e leptospirose) que acometem as cidades brasileiras anualmente (ver detalhes em PACHeCO et al., 2010).

Em adição, a urbanização altera a dinâmica das interações tróficas dos morcegos, e o aumento da abundância de uma dada espécie de determinada guilda alimentar pode indicar a disponibilidade deste item no ambiente (Russo \& AnCilotto, 2015). No presente estudo, mais de $70 \%$ dos dados foram provenientes de duas espécies insetívoras $(M$. molossus e M. nigricans) e uma nectarívora (G. soricina), sendo também as mais frequentes na cidade de São Paulo (Almeida et al., 2015). Estudos prévios relataram que cidades brasileiras beneficiam morcegos generalistas insetívorosfitófagos (PACHECO et al., 2010; Nunes et al.,2016). Um estudo preditivo demonstrou que espécies insetívoras forrageadores de áreas abertas (e.g., Molossus spp.) detém traços ecológicos que melhor explicam a tolerância das espécies em habitats urbanos da América do Sul (Jung \&Threlfall, 2018). De fato, M. molossus é uma das espécies mais abundantes nas cidades brasileiras (PACHECO et al., 2010) e outra espécie de Myotis (M. lucifugus) também já apresentou alta dominância na cidade de Calgary, Canadá (COLEMAN \& BARClay, 2012), reforçando que espécies de Myotis também podem prosperar em meio urbano de regiões tropicais e temperadas. Além disso, nas cidades, humanos determinam a diversidade e densidade das plantas, geralmente selecionadas pela estética paisagística e acabam favorecendo espécies de morcegos oportunistas (BREDT et al., 2012) como as três mais frequentes aqui supracitadas, sem exceção à G. soricina. Apesar desta última ser comumente classificada como nectarívora, estudos prévios demonstraram uma dieta onívora altamente oportunista (incluindo néctar, pólen, frutos e insetos), com marcada variação sazonal e espacial dos itens consumidos (ZorTÉA, 2003; Clare et al., 2014). Considerando o seu oportunismo, a presença de plantas exóticas ornamentais que florescem o ano todo, bem como o hábito dos humanos ofertarem "bebedouros" com solução açucarada para aves deve ser uma fonte alimentar adicional para $G$. soricina, como relatado aos nectarívoros do estado do Arizona, EUA (K. E. Hinman, dados não publicados) incorrendo no aumento da abundância dos morcegos nos locais próximos a fonte alimentar artificial, segundo experimento realizado no Equador (MAGUIÑa \& MuchHala, 2017).

Desse modo, este trabalho demonstra que resultados de monitoramento de quirópteros em longo prazo realizado pelos CCZs corroboram com estudos prévios que documentaram alta dominância de poucas espécies generalistas (JUNG \&KalKo, 2011; Jung \&Threlfall, 2018), as quais se beneficiam da arquitetura e paisagismo das cidades (BREDT et al., 2012; BiavatTi et al., 2015), sendo reflexo do potente filtro biológico não-natural que a urbanização impõe sobre a fauna local (Sol et al., 2014; Nunes et al., 2016). Aqui, provavelmente a habilidade de ocupar os distintos nichos antropogênicos fizeram com que apenas 10,3\% (das 29 spp. aqui registradas) ou 7,5\% (das $40 \mathrm{spp}$. compiladas) aumentassem suas abundâncias e prosperassem em Guarulhos, tornando-as moradoras urbanas ("urban-dwellers") de acordo com a classificação proposta por FisCHER et al. (2015).

Assim, a atuação de equipes multidisciplinares pode promover um efetivo manejo ambiental a fim de reduzir oferta de abrigos em edificações humanas e diminuir a dominância de poucas espécies. Para mitigar o impacto da urbanização sobre a quiropterofauna, PACHECO et al. (2010) recomendam a instalação de abrigos artificiais e aumentar o uso de espécies vegetais provedoras de alimentos para morcegos fitófagos em planos de recuperação de áreas degradadas. Ainda, estes autores alertam para que estas medidas sejam implementadas em locais sem trânsito de pessoas. Isto evita conflitos adicionais entre humanos e morcegos, bem como pode ser utilizado como ferramenta para sensibilizar a população sobre a importância dos morcegos na manutenção dos ecossistemas (ReIs et al., 2007; Boyles et al., 2011; AlmeIDA et al., 2015). Desse modo, o planejamento ambiental das cidades com a inclusão de diretrizes urbanísticas e florísticas torna-se uma interessante ferramenta ao manejo integrado da fauna silvestre e saúde pública, além de aumentar o potencial de conservação da biodiversidade nas cidades e incrementar seus valorosos serviços ecossistêmicos.

Agradecimentos. Nós agradecemos aos funcionários das instituições (Centro de Controle de Zoonoses (CCZ) - São Paulo, CCZ - Guarulhos, Zoológico Municipal de Guarulhos) envolvidos no manejo de morcegos realizados no Município de Guarulhos, sempre dispostos a atender a demanda da população.

\section{REFERÊNCIAS}

Almeida, M. F.; Martorelli, L. F. A.; Sodré, M. M.; Kataoka, A. P. A. G.; Rosa, A. R.; Oliveira, M. L. \& Amatuzzi, E. 2011. Rabies diagnosis and serology in bats from the State of São Paulo, Brazil. Revista da Sociedade Brasileira de Medicina Tropical 44:140-145. Almeida, M. F.; Rosa, A. R.; Sodré, M. M.; Martorelli, L. F. A. \& Trezza Netto, J. 2015. Fauna de morcegos (Mammalia, Chiroptera) e a ocorrência de vírus da raiva na cidade de São Paulo, Brasil. Veterinária e Zootecnia 22(1):89-100.

Alvares, C. A.; Stape, J. L.; Sentelhas, P. C.; Gonçalves, J. L. M. \& SPARoveK, G. 2013. Köppen's climate classification map for Brazil. Meteorologische Zeitschrift 22(6):711-728.

Biavatti, T.; Costa, L. M. \&Esbérard, C. E. L. 2015. Morcegos (Mammalia, Chiroptera) em refúgios diurnos artificiais na região sudeste do Brasil. Mastozoología Neotropical 22(2):239-253.

Boyles, J. G.; Cryan, P. M.; McCracken, G. F. \& Kunz, T. H. 2011. Economic importance of bats in agriculture. Science 332:41-42.

BredT, A. \& UiedA, W. 1996. Bats from urban and rural environments of the Distrito Federal, mid-western Brazil. Chiroptera Neotropical 2(2):54-57.

Bredt, A.; Uieda, W. \& Pedro, W. A. 2012. Plantas e morcegos na recuperação de áreas degradadas e na paisagem urbana. Brasília, Rede de sementes do Cerrado. 273p.

Cabral, A. D.; Gama, A. R. Sodré, M. M.; Savani, E. S. M. M.; GalvãoDias, M. A.; Jordẽo, L. R.; Maeda, M. M.; Yai, L. E. O.; Gennari, S. M. \& PenA, H. F. J. 2013. First isolation and genotyping of Toxoplasma gondii from bats (Mammalia: Chiroptera).Veterinary Parasitology 193:100-104.

Chaves, M. E.; Uieda, W.; Bolochio, C. E.; Souza, C. A. I.; Braga, D. A.; Ferreira, C. H.; Firmo, C. L.; Mariano, R. G. G. C.; Oliveira, K. C. 
S.; SAntos, E. G. \& Costa, F. M. 2012. Bats (Mammalia: Chiroptera) from Guarulhos, state of São Paulo, Brazil. Check List 8:11171121.

Clare, E. L.; Goerlitz, H. R.; Drapeau, V. A.; Holderied, M. W.; Adams, A. M.; Nagel, J.; Dumont, E. R.; Hebert, P. D. N. \& Fenton, B. M. 2014. Trophic niche flexibility in Glossophaga soricina: How a nectar seeker sneaks an insect snack. Functional Ecology 28:632-641. http:// onlinelibrary.wiley.com/doi/10.1111/1365-2435.12192.

COLEMAN, J. L. \& BARCLAY, R. M. R. 2012. Urbanization and the abundance and diversity of Prairie bats. Urban Ecosystems 15:87-102. https:// doi.org/10.1007/s11252-011-0181-8

De Lucca, T.; Rodrigues, R. C. A.; Castagna, C.; Pressotto, D.; De Nadai, D. V.; Fagre, A.; Braga, G. B.; Gullloux, A. G. A.; Alves, A. J. S. e A.; Martins, C. M.; AmakU, M.; Ferreira, F. \& Dias, R. A. 2013. Assessing the rabies control and surveillance systems in Brazil: An experience of measures toward bats after the halt of massive vaccination of dogs and cats in Campinas, Sao Paulo. Preventive Veterinary Medicine 111(1-2):126-133.

Devictor, V.; Julliard, R.; Clavel, J.; Jiguet, F.; Lee, A. \& Couvet, D. 2008. Functional biotic homogenization of bird communities in disturbed landscapes. Global Ecology and Biogeography 17:252-261.

Dormann, C. F.; Gruber, B. \& Fruend, J. 2008. Introducing the bipartite Package: Analysing Ecological Networks. R news 8(2):8-11.

Evelyn, M. J.; Stiles, D. A. \& Young, R. A. 2004. Conservation of bats in suburban landscapes: roost selection by Myotis yumanensis in a residential area in California. Biological Conservation 115:463-473.

Favoretto, S.R.; Carrieri, M. L.; Cunha, E. M. S.; Aguiar, E. A. C., Silva, L. H. Q.; Sodré, M. M.; Souza, M. C. A. M. \& Kotait, I. 2002. Antigenic typing of Brazilian rabies virus samples isolated from animals and humans,1989-2000. Revista do Instituto de Medicina Tropical de São Paulo 44(2):91-95

Fischer, J. D.; SChNeider, S. C.; Ahlers, A. A. \& Miller, J. R. 2015. Categorizing wildlife responses to urbanization and conservation implications of terminology. Conservation Biology 29(4):1246-1248.

Fundação SOS Mata AtLÂNTICA \& INPE - Instituto NaCional DE PESQUisAs ESPACIAIS. 2016. Atlas dos remanescentes florestais da Mata Attântica 2014-2015. São Paulo. Disponível em $<$ https://www. sosma.org.br>. Acesso em 06.01.2019.

GaRBINO, G. S. T. 2016. Research on bats (Chiroptera) from the state of São Paulo, southeastern Brazil: annotated species list and bibliographic review. Arquivos de Zoologia 47(3):43-128.

Gardner, A. L. ed. 2007. Mammals of South America. Marsupials, xenarthrans, shrews, and bats. Vol. 1. London, The University of Chicago Press. 690p.

GREGORIN, R. \&TADDEI, V. A. 2002. Chave artificial para a identificação de Molossídeos brasileiros (Mammalia, Chiroptera). Mastozoología Neotropical 9(1):13-32.

Hourigan, C. L.; Johnson, C. \& Robson, S. K. A. 2006. The structure of a micro-bat community in relation to gradients of environmental variation in a tropical urbanarea. Urban Ecosystems 9:67-82. http:// dx.doi.org/10.1007/s11252-006-7902-4.

Hourigan, C. L.; Catterall, C. P.; Jones, D. \& Rhodes, M. 2010. The diversity of insectivorous bat assemblages among habitats within a subtropical urban landscape. Austral Ecology 35:849-857. http:// dx.doi.org/10.1111/j.1442-9993.2009.02086.x.

IBGE - Instituto Brasileiro de Geografia e Estatistíca. 2010. Mapas. Bases cartográficas dos limites dos municípios brasileiros. Disponível em $<$ https://www.ibge.gov.br $>$. Acesso em 06.05.2019.

IBGE - Instituto Brasileiro de Geografia e Estatistíca. 2018. Cidades e estados. Área territorial e censo populacional do município de Guarulhos. Disponível em <https://www.ibge.gov.br>. Acesso em 04.06.2019.

IUCN. 2019. The IUCN red list of threatened species. Disponível em $<$ https://www.iucnredlist.org/>. Acesso em 06.05.2019.

JUNG, K. \& KALKO, E. K. V. 2011. Adaptability and vulnerability of high flying Neotropical aerial insectivorous bats to urbanization. Diversity and Distributions 17:262-274.

Jung, K. \& Threlfall, C. G. 2018. Trait-dependent tolerance of bats to urbanization: a global meta-analysis. Proceedings of the Royal Society B 285:20181222. http://dx.doi.org/10.1098/rspb.2018.1222.
KolodinsKy, J. \& AleOnG, J. 1990. An integrated model of consumer complaint action applied to services: a pilot study. Journal of Consumer Satisfaction, Dissatisfaction and Complaint Behavior 3:61-70.

Krester, H. E.; Sullivan, P. J. \& KNuth, B. A. 2008. Housing density as an indicator of spatial patterns of reported human-wildlife interactions in Northern New York. Landscape and Urban Planning 84:282-292.

Kunz, T. H.; Torrez, E. B.; Bauer, D.; Lobova, T. \& Fleming, T. H. 2011. Ecosystem services provided by bats. Annals of the New York Academy of Sciences 1223:1-38.

LI, H. \& WiLKIns, K. T. 2015. Selection of building roosts by Mexican free-tailed bats (Tadarida brasiliensis) in an urban area. Acta Chiropterologica 17(2):321-330.

Lourenço-de-Moraes, R.; Malagoli, L. R.; Gerra, V.; Ferreira, R. B.; Affonso, I. P.; Haddad, C. F. B.; Sawaya, R. J. \& Bastos, R. P. 2018. Nesting patterns among Neotropical species assemblages: can reserves in urban areas be failing to protect anurans? Urban Ecosystems 21(5):933-942.

MacGregor-Fors, I.; Morales-Pérez, L. \& Schondube, J. E. 2012. From forests to cities: effects of urbanization on tropical birds. In: LEPCZYK, C. A. \& WARREN, P. S. eds. Urban bird ecology and conservation. Studies in Avian Biology (no. 45). Berkeley, University of California Press, p. 33-48.

Maguiña, R. \& Muchinala, N. 2017. Do artificial nectar feeders affect bat-plant interactions in an Ecuadorian cloud forest? Biotropica 49(5):586-592.

MCKINNEY, M. L. 2006. Urbanization as a major cause of species homogenization. Biological Conservation 127:247-260.

MCKINNEY, M. 2008. Effects of urbanization on species richness: a review of plants and animals. Urban Ecosystem11:161-176.

MinISTÉRIO DA SAÚDE [MS]. 2005. Guia de vigilância epidemiológica, 6ed. Brasília, Ministério da Saúde. 816p.

Myers, N.; Mittermeier, R. A.; Mittermeier,C. G.; Da Fonseca, G. A. B. \& KENT, J. 2000. Biodiversity hotspots for conservation priorities. Nature 403:853-858.

Nogueira, M. R.; Lima, I. P.; Garbino, G. S. T.; Moratelli, R.; Tavares, V. C.; Gregorin, R. \& Peracchi, A. L. 2018. Updated checklist of Brazilian bats: version 2018.1. Comitê da Lista de Morcegos do Brasil - CLMB. Sociedade Brasileira para o Estudo de Quirópteros (Sbeq). $<$ http://www.sbeq.net/updatelist> Acesso em:15.03.2019.

Nunes, H.; RochA, F. L. \& CoRDEIRO-Estrela, P. 2016. Bats in urban areas of Brazil: roosts, food resources and parasites in disturbed environments. Urban Ecosystem 20:953-969.

Oksanen, J.; Blanchet, F. G.; Friendly, M.; Kindt, R.; Legendre, P.; McGlinn, D.; Minchin, P. R.; O’Hara, R. B.; Simpson, G. L.; Solymos, P.; Stevens, M. H. H.; Szoecs, E. \& Wagner, H. 2017. vegan: Community EcologyPackage. R packageversion 2.5-4. https:// CRAN.R-project.org/package=vegan.

Olden, J. D.; Poff, N. L.; Douglas, M. R.; Douglas, M. E. \& Fausch, K. D. 2004. Ecological and evolutionary consequences of biotic homogenization. Trends in Ecology and Evolution 19:18-24.

Pacheco, S. M.; Sodré, M.; Gama, A. R.; Bredt, A.; Cavallini-Sanches, E. M.; Marques, R. V.; Guimarães, M. M. \& Bianconi, G. 2010. Morcegos urbanos: Status do conhecimento e plano de ação para a conservação no Brasil. Chiroptera Neotropical 16:630-647.

Passos, F. C.; Miranda, J. M.; Bernardi, I. P.; KakU-Oliveira, N. Y. \& Munster, L. C. 2010. Morcegos da região sul do Brasil: análise comparativa da riqueza de espécies, novos registros e atualizações nomenclaturais (Mammalia, Chiroptera). Iheringia, Série Zoologia 100:5-34.

Reis, N. R.; Peracchi, A. L.; Pedro, W. A. \& Lima, I. P. eds. 2007. Morcegos do Brasil. Londrina, Universidade Estadual de Londrina. 253p.

Russo, D. \& ANCillotto, L. 2015. Sensitivity of bats to urbanization: a review. Mammalian Biology 80:205-212.

Silva, M. M. S.; Harmani, N. M. S.; GonçalVes, E. F. B. \& Uieda, W. 1996. Bats from metropolitan region of São Paulo, Southeastern Brazil. Chiroptera Neotropical 2(1):39-41.

Sol, D.; González-Lagos, C.; Moreira, D.; Maspons, J. \& LaPiedra, O. 2014. Urbanisation tolerance and the loss of avian diversity. Ecology Letters 17(8):942-950. 
Souza, C. S. A.; Teixeira, C. P. \& Young, R. J. 2012. The welfare of an unwanted guest in an urban environment: the case of the white-eared opossum (Didelphis albiventris). Animal Welfare 21:177-183.

SÃo PAULO. 2018. Decreto n ${ }^{\mathbf{0}}$ 63.853, de 27 de novembro de 2018. Declara as espécies da fauna silvestre no Estado de São Paulo regionalmente extintas, as ameaçadas de extinção, as quase ameaçadas e as com dados insuficientes para avaliação, e dá providências correlatas. Disponível em $<$ https://www.al.sp.gov.br>. Acesso em 21.6.2019.
Uieda, W.; Harmani, N. M. S. \& Silva, M. M. S. 1995. Raiva em morcegos insetívoros (Molossidae) do sudeste do Brasil. Revista de Saúde Pública 29(5):393-397.

Vizotto, L. D. \& TAdDeI, V. A. 1973. Key for determination of Brazilian bats. Journal of the Faculty of Philosophy, Sciences of São José do Rio Preto 1:1-72.

ZorTÉA, M. 2003. Reproductive patterns and feeding habits of three nectarivorous bats (Phyllostomidade: Glossophaginae) from the Brazilian Cerrado. Brazilian Journal of Biology 63(1):159-168. 